\title{
Metabolic engineering of riboflavin production in Ashbya gossypii through pathway optimization
}

\author{
Rodrigo Ledesma-Amaro, Cristina Serrano-Amatriain, Alberto Jiménez and José Luis Revuelta*
}

\begin{abstract}
Background: The industrial production of riboflavin mostly relies on the microbial fermentation of flavinogenic microorganisms and Ashbya gossypii is the main industrial producer of the vitamin. Accordingly, bioengineering strategies aimed at increasing riboflavin production in A. gossypii are highly valuable for industry.

Results: We analyze the contribution of all the RIB genes to the production of riboflavin in A. gossypii. Two important metabolic rate-limiting steps that limit the overproduction of riboflavin have been found: first, low mRNA levels of the RIB genes hindered the overproduction of riboflavin; second, the competition of the AMP branch for purinogenic precursors also represents a limitation for riboflavin overproduction. Thus, overexpression of the RIB genes resulted in a significant increase in riboflavin yield. Moreover, both the inactivation and the underexpression of the ADE12 gene, which controls the first step of the AMP branch, also proved to have a positive effect on riboflavin production. Accordingly, a strain that combines both the overexpression of the RIB genes and the underexpression of the ADE12 gene was engineered. This strain produced $523 \mathrm{mg} / \mathrm{L}$ of riboflavin (5.4-fold higher than the wild-type), which is the highest titer of riboflavin obtained by metabolic engineering in A. gossypii so far.
\end{abstract}

Conclusions: Riboflavin production in A. gossypii is limited by a low transcription activity of the RIB genes. Flux limitation towards AMP provides committed substrate GTP for riboflavin overproduction without detrimental effects on biomass formation. A multiple-engineered Ashbya strain that produces up to $523 \mathrm{mg} / \mathrm{L}$ of riboflavin was generated.

Keywords: Riboflavin, Ashbya gossypii, RIB, ADE12, Metabolic engineering

\section{Background}

Riboflavin (vitamin B2) is an essential nutrient for humans and animals that must be obtained from the diet. Accordingly, it is usually included in fortified foods and multivitamin supplements [1]. The production of riboflavin by microbial fermentation is a paradigmatic example of white biotechnology using different cell factories such as Bacillus subtilis, Candida flareri and, especially, Ashbya gossypii [2]. Indeed, nearly half the world production of riboflavin (8000 tons per year) is obtained through the fermentation of $A$. gossypii strains [3].

Ashbya gossypii is a filamentous hemiascomycete that overproduces riboflavin naturally. Thus, industrial

\footnotetext{
*Correspondence: revuelta@usal.es

Departamento de Microbiología y Genética, Universidad de Salamanca, Campus Miguel de Unamuno, 37007 Salamanca, Spain
}

strains of A. gossypii for riboflavin production have been obtained by a combination of random chemical mutagenesis with metabolic engineering of the purine pathway [1]. The development of rational approaches for the manipulation of $A$. gossypii is now supported with novel genomic tools, including a genome-scale metabolic model of A. gossypii (iRL766) [4]. In addition, new strains of $A$. gossypii for the production of folic acid and singlecell oil have been described recently $[5,6]$.

The biosynthesis of riboflavin is initiated from two main precursors: GTP and ribulose-5-phosphate (Ribu5P) (Fig. 1). GTP is synthesized from the purine pathway and its availability has been shown to be important for riboflavin production. In this regard, the increase in metabolic flux towards the synthesis of GTP results in the overproduction of riboflavin [7-9]. GTP and Ribu5P 


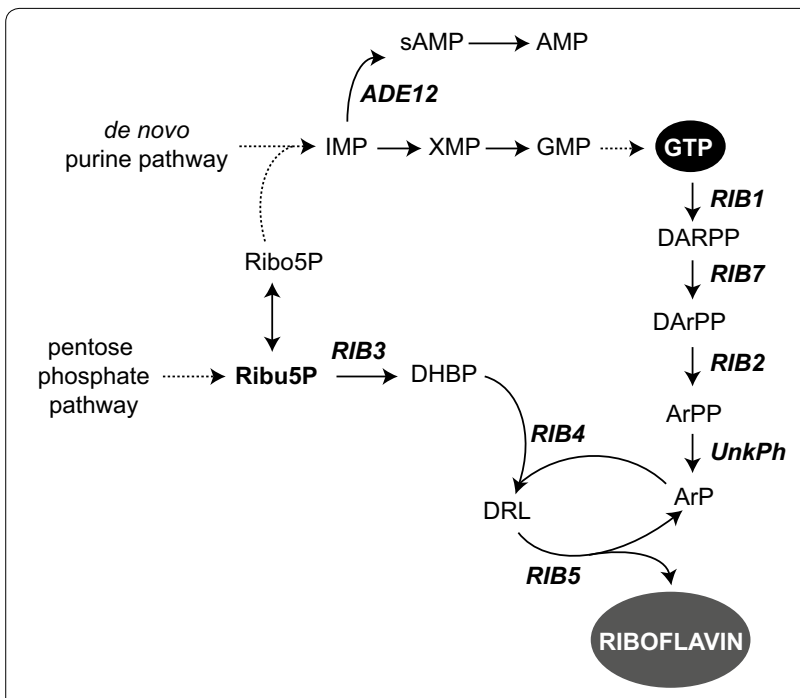

Fig. 1 Diagram of the riboflavin biosynthetic pathway. The genes manipulated in this work are indicated in bold. Dashed lines indicate multistep reactions. Ribu5P ribulose-5-phosphate; ribo5P ribose-5-phosphate; DARPP 2,5-diamino-6-ribosylamino4(3H)-pyrimidinedione phosphate; DArPP 2,5-diamino-6ribitylamino-4-(3H)-pyrimidinedione-5'-phosphate; ArPP 5-amino-6-ribitylamino-2,4-(1H,3H)-pyrimidinedione-5'-phosphate; ArP 5-amino-6-ribitylamino-2,4-(1H,3H)-pyrimidinedione; DHBP 3,4-dihydroxy-2-butanone-4-phosphate; $D R L$ 6,7-dimethyl-8-ribityllumazine

are transformed into riboflavin via a 7-reaction pathway that is controlled by the RIB genes [10] (Fig. 1). There are two branches that converge to form 6,7-Dimethyl-8-ribityl-lumazine (DRL). One branch synthesizes 5-Amino6-ribitylamino-2,4- $(1 H, 3 H)$-pyrimidinedione (ArP) from GTP through four consecutive reactions catalyzed by the enzymes RIB1, RIB7, RIB2 and an unknown phosphatase (Fig. 1). On the other branch, RIB3 transforms Ribu5P into 3,4-Dihydroxy-2-butanone-4-phosphate (DHBP). Then, both ArP and DHBP are conjugated by RIB4 to produce DRL, which is subsequently converted into riboflavin by the product of the RIB5 gene (Fig. 1). All the RIB genes have been identified in $A$. gossypii [11] but so far their contribution to riboflavin overproduction has not been fully characterized.

In A. gossypii riboflavin is mainly produced during the production phase, when the growth rate decreases. In contrast, exponential growth occurs during the trophic phase, when riboflavin yield is minimal [10]. Accordingly, riboflavin biosynthesis is tightly regulated at both the enzymatic and transcriptional levels as previously described both for the purine and RIB pathways in $A$. gossypii $[7,9,11]$. In this regard, transcriptional regulation occurred during the riboflavin production phase has been predicted by a genome-scale metabolic reconstruction, thus revealing a number of target genes that can be considered as good candidates for metabolic engineering approaches to increase the production of riboflavin. Interestingly, the RIB2, RIB3, RIB4 and RIB5 genes were predicted to be up-regulated genes during riboflavin overproduction in A. gossypii [4]. In good agreement, several reports have also shown a relationship between the transcriptional regulation of $R I B$ genes and an increase of the riboflavin biosynthesis in several organisms [12-14].

As mentioned above, riboflavin overproduction has been achieved through the deregulation of the purine pathway at different levels to increase the pool of GTP [7-9]. It is noteworthy that both the GMP and AMP branches of the purine pathway compete for the common precursor IMP. Thus, in C. famata (C. flareri), the heterologous expression of both the transcription factor SEF1 and the purinogenic enzyme IMP dehydrogenase from Debaryomyces hansenii, together with the overexpression of the RIB1 and RIB7 genes, resulted in a 4.1-fold increase in riboflavin production [15]. Hence, a limiting riboflavin biosynthetic pathway may mask the identification of new mutations improving the production of riboflavin.

The objective of this work was to study the relative contribution of each $R I B$ gene to the riboflavin production process in A. gossypii. Accordingly, the transcriptional pattern of the $R I B$ genes was analyzed and the potential impact of each RIB gene on the metabolic control of riboflavin production was determined by overexpression of single $R I B$ genes as well as by combining the overexpression of up to five RIB genes to deregulate the entire pathway. In addition, the performance of a deregulated strain overexpressing the full set of $R I B$ genes was challenged to support higher riboflavin productivity in response to a major availability of GTP substrate. This was achieved by metabolic flux re-direction towards GMP by reducing the synthesis of AMP from IMP. In summary, we found that both the availability of GTP substrate from the purine pathway and the transcriptional activity of the RIB genes are rate-limiting steps for riboflavin overproduction.

\section{Results}

Transcriptional analysis of the RIB genes in A. gossypii

As previously described, several reports have established a relationship between the transcriptional regulation of some of the RIB genes and the increase of riboflavin production [11-14]. In addition, the analysis of metabolic flux changes related to the production of riboflavin using the iRL766 model predicted the up-regulation of most of the RIB genes during the production phase in A. gossypii [4]. Accordingly, we were prompted to determine the RNA levels of all the six $R I B$ genes encoded in the genome of $A$. gossypii.

The transcriptional analysis of the $R I B$ genes was carried out by qRT-PCR. Total RNA from A. gossypii MA2 
cultures in both the trophic phase (24 h of culture) and the production phase (120 h of culture) were obtained. Quantitative analysis of the RIB genes in cDNA samples was carried out using the housekeeping gene ACT1 as a reference [6]. With the exception of RIB4, the level of RNA for all the RIB genes was very low in both the trophic and production phases; ranging from 1-5 (for RIB2, RIB5 and RIB7) to $10-15$ \% (for RIB1 and RIB3) of the transcript level of the moderately transcribed gene ACT1 (Fig. 2). RIB4 showed a high transcriptional level, similar to that of the GPD gene (Fig. 2), whose promoter is widely used for gene overexpression in A. gossypii [9]. In contrast to the prediction of the iRL766 model, the transcription rate of the RIB genes showed no or only minor increments during the production phase (Fig. 2).

\section{Single gene overexpression of the RIB genes in A. gossypii}

The results reported above revealed that the transcription of most of the RIB genes remains low throughout the trophic and production phases of $A$. gossypii culture. Accordingly we wished to determine the effect of the overexpression of each $R I B$ gene on riboflavin production in A. gossypii. The RIB4 was excluded from the overexpressions experiments, since this gene already showed a high transcriptional level in the wild-type.

Gene overexpressions were carried out using an integrative cassette harboring a geneticin-resistance selection marker and the strong promoter $P_{A g G P D}$. The cassette was flanked by recombinogenic sequences for each $R I B$ gene and was integrated by homologous recombination

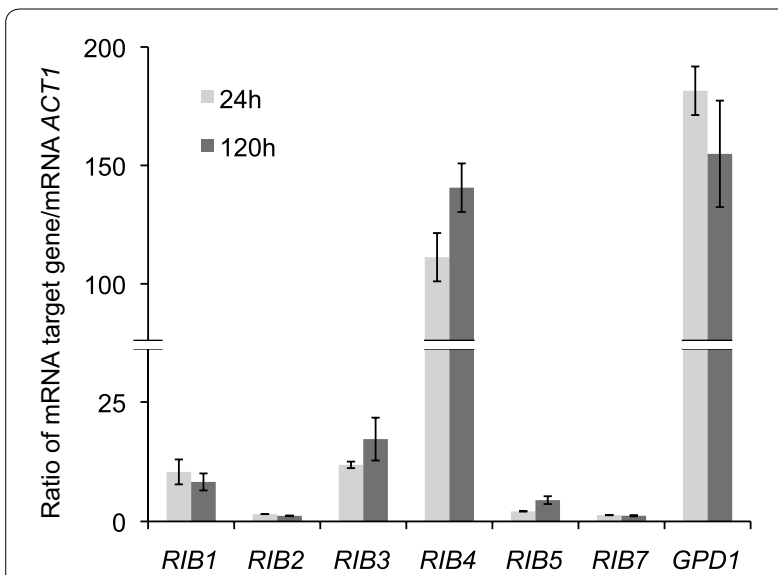

Fig. 2 Quantitative-real time PCR of the RIB genes in A.gossypii. Relative transcription levels of the RIB genes in our wild-type strain of A. gossypii during both the trophic phase ( $24 \mathrm{~h}$, exponential growth) and the production phase (120 h, stationary growth). The GPD1 gene was included as a control. Transcription levels were normalized using the A. gossypii ACT1 gene as a reference. The results are the means of two independent experiments performed in duplicate and are expressed as a ratio of the cDNA abundances of the target genes with respect to the ACT1 mRNA levels to replace the native promoter region of each gene. Overexpression of the RIB genes was verified by qRT-PCR analysis (Additional file 1: Fig. S1a) and the riboflavin production of the mutant strains was determined. As shown in the Fig. 3, the overexpression of RIB1, RIB2 or $R I B 3$ triggered an increase in riboflavin production. The overexpression of either $R I B 1$ or $R I B 3$, which code for the first enzymes of each branch in the riboflavin pathway, induced the highest increases in riboflavin production. In contrast, the overexpression of either RIB5 or RIB7 did not cause any significant change in the production of riboflavin (Fig. 3).

\section{Global overexpression of the RIB genes in A. gossypii}

We observed that overexpression of some of the RIB genes induced an increase in riboflavin production in A. gossypii. Thus, the low transcription rate of the $R I B$ genes may be a limiting-rate step for riboflavin overproduction and, consequently, a maximal yield of riboflavin might be obtained with the overexpression of all the RIB genes. Accordingly, it was decided to overexpress all the $R I B$ genes to quantify the riboflavin production in a quintuple overexpressing strain. A loxP-KanMX-loxP selection marker was used for gene overexpression. The loxP repeated inverted sequences enabled the selection marker to be eliminated, and subsequently reused, by expressing a Cre recombinase after each round of transformation, as described elsewhere [6]. The gene overexpression pipeline is depicted in Fig. 4a. The A329 strain, which overexpresses the RIB1, RIB2, RIB3, RIB5 and RIB7 genes, was obtained after 10 transformations either to integrate the $P_{A g G P D}$ into the target loci or to remove the $\operatorname{KanMX}$ selection marker (Fig. 4a). The overexpression of the RIB genes in the new mutant strains was verified by qRT-PCR (Additional file 1: Fig. S1b).

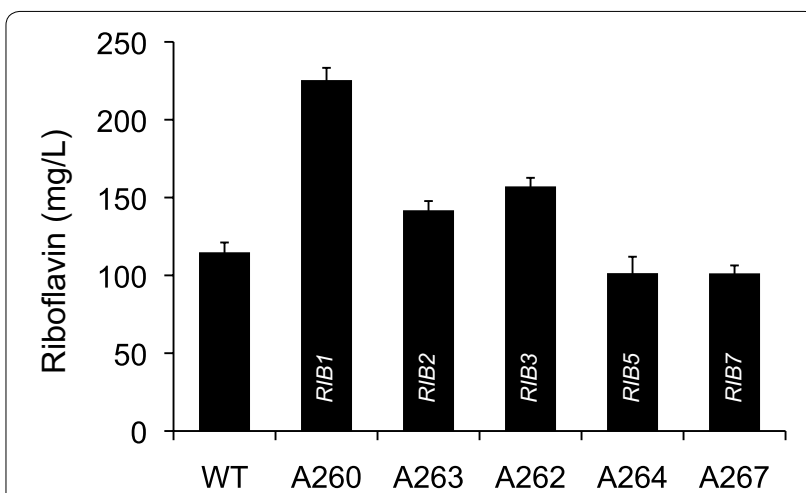

Fig. 3 Riboflavin production after the overexpression of RIB genes in A. gossypii. Quatification of riboflavin production in the A. gossypii strains that overexpress one of the RIB genes. The data are the means of three independent experiments performed in duplicate 
a

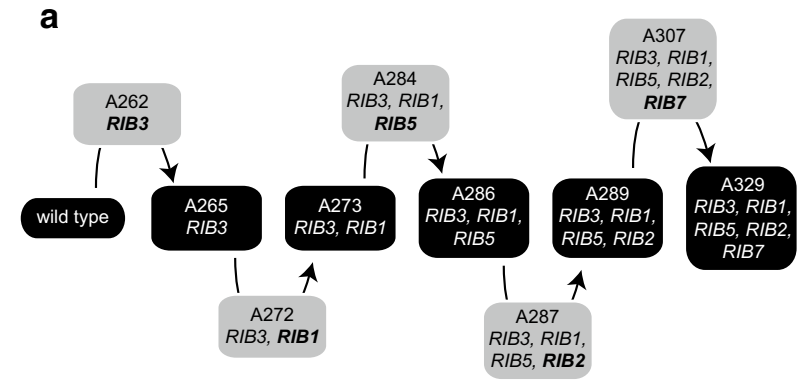

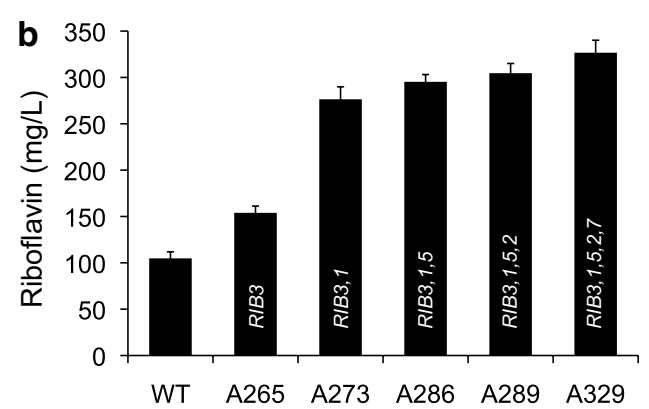

Fig. 4 Overexpression of the RIB genes in a single strain of A. gossypii. a Pipeline of the construction of the A329 strain and $\mathbf{b}$ quatification of riboflavin production in the A. gossypii strains. The data are the means of three independent experiments performed in duplicate

Riboflavin production was measured in the quintuple overexpressing strain and also in all the intermediate strains (Fig. 4b). As shown above, the overexpression of RIB3 alone triggered a remarkable increase in riboflavin production ( $46 \%$ more riboflavin than the WT strain). In addition, the combination of the overexpression of both RIB1 and RIB3 (strain A273) increased the riboflavin yield by more than 2.5-fold in comparison with the WT. Moreover, the overexpression of RIB5, RIB2 and RIB7 caused an additional increase in riboflavin production with respect to the A273 strain. Overall, the A329 strain, which simultaneously overexpressed the RIB1, RIB2, RIB3, RIB5 and RIB7 genes, showed the highest level of riboflavin production $(326.6 \mathrm{mg} / \mathrm{L})$, representing a 3.1-fold increase over the WT production (Fig. 4b).

\section{Flux balance analysis of the purine pathway for riboflavin production}

In the absence of enzymatic regulation constrains, the strain containing the overexpressed, deregulated riboflavin biosynthetic pathway should not be limited in the riboflavin pathway itself, but rather it should be able to respond to increases in the pool of the GTP substrate by efficiently increasing the rate of riboflavin product formation. To assess this hypothesis we decided to increase the GTP supply available for riboflavin synthesis and determine the effect on riboflavin production in both the WT and RIB-overexpressing (A329) strains.

The purine pathway provides GTP, which is one of the main precursors of riboflavin. Since our previous results demonstrated that the deregulation of the purine pathway induces a significant increase of riboflavin overproduction in A. gossypii [7-9], an increase in the intracellular pool of GTP could be correlated with a higher riboflavin yield in A. gossypii. IMP is the central metabolite of the purine pathway, which can subsequently be converted either to AMP or GMP via a two-branch pathway (Fig. 1). Thus, IMP metabolic flux towards GMP/GTP production compete with the AMP branch, which consists of two enzymatic reactions controlled by adenylosuccinate synthase $(A D E 12)$ and adenylosuccinate lyase $(A D E 13)$. Indeed, flux balance analysis of riboflavin production using the $A$. gossypii iRL766 model predicted that the AMP branch of the purine pathway is down-regulated during the production phase, while the GMP branch, leading to GTP and thereafter riboflavin, is not transcriptionally regulated. These results suggest that a control over the IMP hub affects riboflavin production.

Accordingly, we used the A. gossypii iRL766 model to simulate a reduction in metabolic flux towards the AMP branch by decreasing the activity of the Ade12 enzyme (Fig. 1). The in silico simulation comprised 316 metabolic reactions, including enzymatic cofactors that could affect metabolic flux exchange in both the purine and riboflavin pathways. The output of the model predicted a linear increase in riboflavin production that was directly correlated with the reduction in Ade12 activity (Additional file 2: Fig. S2). Thus, in order to increase the supply of GTP available for riboflavin synthesis in A. gossypii, the $A D E 12$ gene can be considered a solid candidate for metabolic engineering through deletion. In addition, engineering the IMP metabolic hub can serve to analyze the performance of a deregulated, overexpressed riboflavin pathway in response to a greater availability of guanine precursors.

\section{Metabolic engineering of the IMP hub gene in A. gossypii}

To analyze the effect of the $A D E 12$ gene-deletion on riboflavin production in $A$. gossypii a gene knockout strain was constructed using a loxP-KanMX-loxP selection marker flanked by $A D E 12$-flanking recombinogenic sequences, as described above (see "Methods" section). As expected, the ade12 $\Delta$ strain was viable but showed adenine auxotrophy. Analysis of riboflavin production 
revealed that the ade $12 \Delta$ strain was able to produce up to $246 \mathrm{mg} / \mathrm{L}$ of riboflavin, which represents a 2.5fold increase in riboflavin production compared to the wild-type strain. In this sense, our results confirm the idea of GTP availability as a limiting step for riboflavin overproduction.

Although the deletion of $A D E 12$ triggers the production of riboflavin, the presence of auxotrophic mutations in the strains is highly undesirable for large-scale industrial fermentations. Therefore, we decided to analyze the effect of $A D E 12$ gene underexpression on riboflavin production. As shown above, the RIB7 gene has very low levels of mRNA in the WT strain. Indeed, a qRT-PCR analysis revealed that the transcription rate of RIB7 was approximately 62-fold lower than that of the $A D E 12$ gene. Accordingly, the promoter sequence of the $R I B 7$ gene $\left(P_{R I B 7}\right)$ was used for $A D E 12$ gene underexpression. Thus, the $A D E 12$ native promoter was replaced by $P_{R I B 7}$ using a loxP-KanMX-loxP selection marker, which was subsequently eliminated as described above (Fig. 5a). The promoter replacement was confirmed both by DNA sequencing of the genomic amplicon (data not shown) and by qRT-PCR analysis. As expected, the mRNA levels of $A D E 12$ were extremely reduced in the $P_{R I B}-A D E 12$ strain (approximately 70-fold lower than those in the wild-type strain). However, this transcription level was sufficient to sustain the growth of the mutant in MA2 media without adenine supplementation. Moreover, the underexpression of $A D E 12$ did not significantly affect biomass formation and riboflavin production was similar to that of the ade $12 \Delta$ strain (Fig. 5b).
Engineering the metabolism of purines and overexpression of the RIB genes trigger riboflavin overproduction

As shown above, the supply of guanine precursors and the transcription of the $R I B$ genes largely determine the riboflavin yield in A. gossypii. Accordingly, in order to analyze the a riboflavin overproducer strain, we decided to combine both the overexpression of the $R I B$ genes and the underexpression of the $A D E 12$ gene.

The quintuple $R I B$-engineered strain A329, which overexpresses the RIB1, RIB2, RIB3, RIB5 and RIB7 genes, was transformed with either the ade12 $\Delta$ deletion cassette or the $P_{R I B 7}$ cassette to replace the $A D E 12$ native promoter. The genomic integrations of each module were confirmed both by analytical PCR and qRT-PCR to test $A D E 12$ mRNA (data not shown). Other parameters, such as the growth rate, biomass production and sporulation ability, were analyzed but no significant differences were found between these new strains and the wild-type strain (data not shown). Finally, a marked increase in riboflavin production was found when these two approaches were conjugated in a single strain (Fig. 6). A total yield of $523 \mathrm{mg} / \mathrm{L}$ of riboflavin was obtained when the overexpression of RIB genes and the underexpression of $A D E 12$ were combined. This riboflavin level in our mutant strain represents an increase of 5.4-fold with respect to production by the wild-type strain (Fig. 6).

\section{Discussion}

Riboflavin production by microbial fermentation is a paradigm of a biotechnological process that has replaced chemical synthesis in the industry. Although different microorganisms are used for the microbial production of
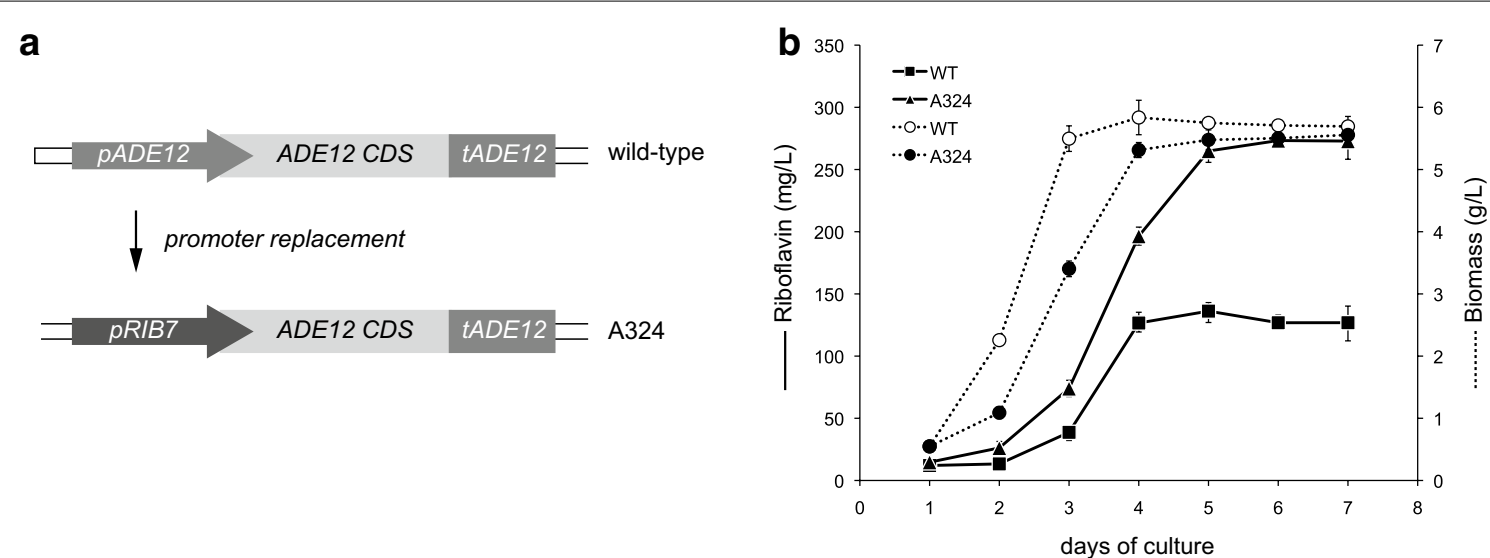

Fig. 5 Underexpression of the ADE12 gene in A. gossypii. a Diagram of the promoter replacement of the ADE12 gene and b biomass and riboflavin production analyses after the underexpression of the ADE12 gene in A. gossypii. The data are the means of three independent experiments performed in duplicate 


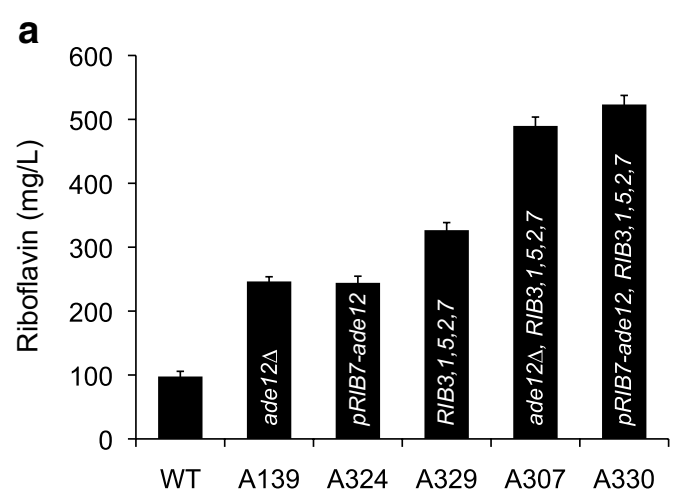

b

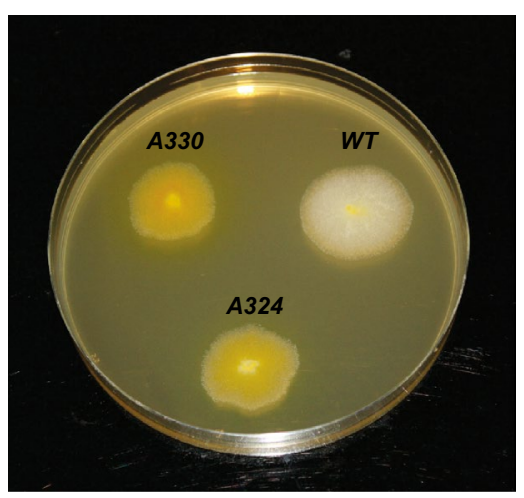

Fig. 6 Riboflavin overproduction in the engineered A. gossypii. a quatification of riboflavin production in A. gossypii strains. The data are the means of three independent experiments performed in duplicate. b solid MA2 plates of the strains A330, A324 and wild-type of A. gossypii. The strain A330 that was modified both for the underexpression of the $A D E 12$ gene and for the overexpression of five RIB genes afforded the highest riboflavin yield (in yellow)

riboflavin, A. gossypii has proved to be one of the most effective producers for industry [2, 16]. Accordingly, the metabolic engineering of $A$. gossypii for riboflavin overproduction represents an important challenge for research in biotechnology.

The transcription of the RIB genes in A. gossypii has been analyzed during both the trophic and production phases. We found that the RIB4 gene was highly transcribed, mainly during the production (stationary) phase, which might indicate the presence of an inducible promoter within the RIB4 sequence. In fact, according to previously published transcriptomic data, RIB4 is the 28th most abundant RNA during the stationary phase in A. gossypii [17]. Therefore, the RIB4 promoter could be an interesting genetic tool for the overexpression of target proteins during the stationary phase in A. gossypii. In contrast to RIB4, the RIB2 and RIB7 genes were very poorly transcribed during $A$. gossypii growth and production phases. Indeed, the substitution of the $A D E 12$ native promoter by the RIB7 promoter was sufficient to minimize the activity of $A D E 12$ and the metabolic flux towards AMP synthesis. This represents proof of concept for the use of the RIB7 promoter for gene underexpression in A. gossypii. This strategy may be very useful as a new molecular tool both to prevent undesired auxotrophies in industrial strains and also to study the effect of the downregulation of essential genes.

We found that overexpression of RIB1 and RIB3 (either single or combined overexpression) triggered a high increase in riboflavin yield, which indicates that the first steps of each branch of the riboflavin biosynthetic pathway (governed by the RIB1 and RIB3 genes) are important control points for riboflavin production. Nevertheless, the quintuple overexpressing strain rendered the highest level of riboflavin production, affording $326.6 \mathrm{mg} / \mathrm{L}$. All together, these results suggest that all the RIB genes are coordinately expressed so that the control of riboflavin production in A. gossypii is more or less evenly distributed over all the genes and enzymes of the RIB pathway and lacks strong controlling individual steps.

It has been previously shown that increasing the pool of metabolic precursors from the purine pathway also induces a significant overproduction of riboflavin in $A$. gossypii $[7-9,18]$. In this regard, an in silico analysis of the biosynthesis of riboflavin using the genome-scale metabolic model iRL766 [4] predicted an increase in riboflavin production by reducing the competing branch leading to AMP synthesis. Accordingly, the deletion or underexpression of $A D E 12$ caused an enhancement of riboflavin accumulation, thus supporting the idea that the availability of the immediate precursor of riboflavin biosynthesis is another metabolic restriction for riboflavin overproduction.

The overexpression of the RIB genes ensures that the cell is devoid of bottlenecks in the riboflavin biosynthetic pathway. This provides a cell model system to identify new mutations or target genes improving riboflavin production that otherwise would be masked by a limiting riboflavin biosynthetic pathway. The overexpression of the riboflavin pathway is also a strategy of great value for further improvement of current industrial strains because it is highly unlikely that those strains may have accumulated all the necessary mutations required for overexpression of all six RIB genes. In this regard, we found that the underexpression of the $A D E 12$ gene has an additive effect over the overexpression of five RIB genes, providing the highest yield of riboflavin production obtained by metabolic engineering in A. gossypii so far. According to our results, metabolic engineering of the IMP hub and improvement of the riboflavin biosynthetic pathway may 
circumvent two important restraints limiting production of riboflavin in A. gossypii. We believe that iterative metabolic engineering in combination with fluxomics could help drive forward both our understanding of riboflavin metabolism in $A$. gossypii and the generation of new strains with a higher riboflavin production capacity.

\section{Conclusions}

We used metabolic engineering to combine the overexpression of all the $R I B$ genes with the enhancement of the GMP purine branch to generate a strain (A330) that produces 5.4-fold more riboflavin than the wild-type strain $(523 \mathrm{mg} / \mathrm{L})$. We foresee that this strategy will provide a scalable and controllable way to increase the industrial production of this economically important vitamin.

\section{Methods}

Ashbya gossypii strains, media, and growth conditions. The A. gossypii ATCC 10895 strain was used and considered a wild-type strain. Other $A$. gossypii strains used in the study are listed in the Additional file 4: Table S2. A. gossypii was cultured at $28{ }^{\circ} \mathrm{C}$ using either MA2 rich medium or synthetic minimal medium lacking adenine (SC-ade) [9]. A. gossypii transformation, its sporulation conditions and spores isolation were as previously described $[9,19]$.

Quantitative Real-Time PCR. Quantitative real-time PCR (qRT-PCR) was performed with a LightCycler 480 real-time PCR instrument (Roche), using SYBR green I master mix (Roche) and following the manufacturer's instructions. Total RNA samples were obtained as described previously [7] and cDNA samples were prepared using the Transcriptor First Strand cDNA Synthesis Kit (Roche). Primer sequences are indicated in the Additional file 4: Table S2. All real-time PCR reactions were performed in duplicate and in at least two independent experiments. Quantitative analyses were carried out using the LightCycler 480 software. Quantitative values were obtained as the threshold PCR cycle number $(C t)$ when the increase in the fluorescent signal of the PCR product showed exponential amplification. The target gene mRNA level was normalized to that of AgACT1 in the same sample. The relative transcription level of the target gene was calculated using the $2^{-\Delta \Delta C t}$ method [20].

Gene overexpression and gene deletion. For gene overexpression $_{A g G P D}$, the promoter sequence of the $A g G P D$ gene was integrated upstream of the ATG initiator codon of each gene. An overexpression cassette comprising the $A g G P D$ promoter $(P)$ and the loxPKanMX-loxP selectable marker, conferring resistance to geneticin (G418), was PCR-amplified using specific primers for each gene (Additional file 4: Table S2). The overexpression modules were used to transform spores of $A$. gossypii and positive clones were selected in G418-containing medium. Homokaryon clones were obtained by sporulation of the primary transformants. The correct genomic integration of each overexpression cassette was confirmed by analytical PCR followed by DNA sequencing. Gene overexpression was checked by qRT-PCR analysis. For the deletion of $A g A D E 12$, a gene replacement cassette was constructed for the $A D E 12$ gene by PCR amplification of the loxP-KanMX-loxP marker (see primer sequences in the Additional file 4: Table S2). The replacement cassette was used to transform spores of A. gossypii. Primary transformants were selected in G418-containing medium and homokaryon clones were isolated by sporulation of the primary transformants. The homologous integration of the replacement cassette was confirmed by analytical PCR followed by DNA sequencing.

Determination of riboflavin production. Riboflavin was measured using a spectrophotometric method. Briefly, a volume of $1 \mathrm{~mL}$ of the culture was harvested and mixed with $1 \mathrm{~mL}$ of $0.1 \mathrm{~N} \mathrm{HCl}$. The suspension was heated at $100{ }^{\circ} \mathrm{C}$ for $30 \mathrm{~min}$. Then, samples were cooled down and mycelia were broken using glass beads. The liquid phase containing both extracellular and intracellular riboflavin was recovered and used for riboflavin determination. A calibration curve was performed using pure riboflavin (Sigma-Aldrich), which was processed in an identical way to the samples. Riboflavin concentration was determined by reading the absorbance of each sample at $450 \mathrm{~nm}$. Measurements were performed using a Varioskan microtiter plate reader (Thermo Scientific).

In silico flux balance analysis. Flux balance analysis calculations were performed using MATLAB (Mathworks, Natick, MA) and the Raven toolbox [21]. The iRL766 genomic scale model for A. gossypii was used [4]. To calculate the maximum theoretical yields, riboflavin production reaction 'RFLAVxtO' was set as the objective function and the system of linear equations was solved. Biomass and glucose uptake were constrained to $0.09 \mathrm{~h}^{-1}$ and $1 \mathrm{mmol} / \mathrm{gh}$, respectively, while $\mathrm{NH} 3$, sulfate, phosphate and adenine remained opened. The flux through ADE12 was fixed to $0.0117 \mathrm{mmol} / \mathrm{gh}$ (the maximum theoretical rate) and reduced manually to simulate a reduction in its metabolic flux. The model simulates adenine utilization from the salvage purine pathway, which allows normal growth even when the de novo pathway for adenine biosynthesis is limited. The on line tool Biomet toolbox 2.0 (http://biomet-toolbox.org/) was used to check the flux balance analysis. 


\section{Additional files}

Additional file 1: Fig. S1. Quantitative-real time $P C R$ of $R / B$ genes in $A$. gossypii mutants. A, relative transcription levels of the $R / B$ genes in the strains containing overexpression modules for each $R / B$ gene compared with the WT strain. B, relative transcription levels of the RIB genes both in the $W T$ and the $A 329$ strains. Transcription levels were normalized using the A. gossypii ACT1 gene as a reference. The results are means of two independent experiments performed in duplicate and are expressed as a ratio of the CDNA abundances of the target genes with respect to the ACT1 mRNA levels.

Additional file 2: Fig. S2. Correlation between the ADE12-catalyzed flux and riboflavin production in A. gossypii. The A. gossypii iRL766 model was used to predict the effect of the ADE12 flux reduction over riboflavin biosynthesis. See "Methods" for details.

Additional file 3: Table S1. A. gossypii strains used in this study.

Additional file 4: Table S2. List of primers used in this study.

\section{Authors' contributions}

RL-A designed and performed most of the experiments. CS-A designed and performed experiments. AJ coordinated the study and wrote the manuscript. $J L R$ conceived the project, designed the experiments and wrote the manuscript, with the significant contribution of all co-authors. All authors read and approved the final manuscript.

\section{Acknowledgements}

This work was supported in part by BASF SE and grant BIO2014-23901 from the Spanish Ministerio de Economía y Competitividad. R. L.-A. and C. S.-A. were recipients of FPU predoctoral fellowships from the Spanish Ministerio de Economía y Competitividad. We thank M. D. Sánchez and S. Domínguez for excellent technical help and N. Skinner for correcting the manuscript.

\section{Competing interests}

RLA, CSA, AJ and JLR declare conflict of interest due to issued and outstanding patent applications covering aspects of this work authors declare that they have no competing interests.

Received: 30 July 2015 Accepted: 4 October 2015

Published online: 14 October 2015

\section{References}

1. Ledesma-Amaro R, Jiménez A, Santos M, Revuelta J. Microbial production of food ingredients, enzymes and nutraceuticals. Microbial production of vitamins, vol. 246. Sawston: Woodhead Publishing Series in Food Science; 2013.

2. Stahmann KP, Revuelta JL, Seulberger $H$. Three biotechnical processes using Ashbya gossypii, Candida famata, or Bacillus subtilis compete with chemical riboflavin production. Appl Microbiol Biotechnol. 2000;53(5):509-16.

3. Park EY, Ito Y, Nariyama M, Sugimoto T, Lies D, Kato T. The improvement of riboflavin production in Ashbya gossypii via disparity mutagenesis and DNA microarray analysis. Appl Microbiol Biotechnol. 2011;91(5):1315-26. doi:10.1007/s00253-011-3325-0.

4. Ledesma-Amaro R, Kerkhoven EJ, Revuelta JL, Nielsen J. Genome scale metabolic modeling of the riboflavin overproducer Ashbya gossypii. Biotechnol Bioeng. 2013; doi:10.1002/bit.25167.
5. Ledesma-Amaro R, Santos M-A, Jiménez A, Revuelta JL. Tuning singlecell oil production in Ashbya gossypii by engineering the elongation and desaturation systems. Biotechnol Bioeng. 2014;111(9):1782-91. doi:10.1002/bit.25245

6. Ledesma-Amaro R, Santos MA, Jiménez A, Revuelta JL. Strain design of Ashbya gossypii for single-cell oil production. Appl Environ Microbiol. 2014;80(4):1237-44. doi:10.1128/AEM.03560-13.

7. Mateos L, Jiménez A, Revuelta JL, Santos MA. Purine biosynthesis, riboflavin production, and trophic-phase span are controlled by a Mybrelated transcription factor in the fungus Ashbya gossypii. Appl Environ Microbiol. 2006;72(7):5052-60.

8. Jiménez A, Santos MA, Revuelta JL. Phosphoribosyl pyrophosphate synthetase activity affects growth and riboflavin production in Ashbya gossypii. BMC Biotechnol. 2008;8:67.

9. Jiménez A, Santos MA, Pompejus M, Revuelta JL. Metabolic engineering of the purine pathway for riboflavin production in Ashbya gossypii. App Environ Microbiol. 2005;71(10):5743-51.

10. Schlosser T, Wiesenburg A, Gatgens C, Funke A, Viets U, Vijayalakshmi $\mathrm{S}$, et al. Growth stress triggers riboflavin overproduction in Ashbya gossypii. Appl Microbiol Biotechnol. 2007;76(3):569-78. doi:10.1007/ s00253-007-1075-9.

11. Revuelta JL, Buitrago MJ, Santos MA, Inventors; BASF AG, Assignee. Riboflavin synthesis in fungi. 1995.

12. Perkins JB, Sloma A, Hermann T, Theriault K, Zachgo E, Erdenberger T, et al. Genetic engineering of Bacillus subtilis for the commercial production of riboflavin. J Ind Microbiol Biotech. 1999;22(1):8-18. doi:10.1038/ sj.jim.2900587.

13. Marx H, Mattanovich D, Sauer M. Overexpression of the riboflavin biosynthetic pathway in Pichia pastoris. Microb Cell Fact. 2008;7:23. doi:10.1186/1475-2859-7-23.

14. Karos M, Vilarino C, Bollschweiler C, Revuelta JL. A genome-wide transcription analysis of a fungal riboflavin overproducer. J Biotechnol. 2004;113(1-3):69-76. doi:10.1016/j.jbiotec.2004.03.025.

15. Dmytruk KV, Yatsyshyn VY, Sybirna NO, Fedorovych DV, Sibirny AA. Metabolic engineering and classic selection of the yeast Candida famata (Candida flareri) for construction of strains with enhanced riboflavin production. Metab Eng. 2011;13(1):82-8. doi:10.1016/j. ymben.2010.10.005

16. Abbas CA, Sibirny AA. Genetic control of biosynthesis and transport of riboflavin and flavin nucleotides and construction of robust biotechnological producers. Microbiol Mol Biol Rev. 2011;75(2):321-60. doi:10.1128/ MMBR.00030-10.

17. Gattiker A, Rischatsch R, Demougin P, Voegeli S, Dietrich FS, Philippsen P, et al. Ashbya Genome Database 3.0: a cross-species genome and transcriptome browser for yeast biologists. BMC Genom. 2007;8:9. doi:10.1186/1471-2164-8-9.

18. Schlupen C, Santos MA, Weber U, de Graaf A, Revuelta JL, Stahmann KP. Disruption of the SHM2 gene, encoding one of two serine hydroxymethyltransferase isoenzymes, reduces the flux from glycine to serine in Ashbya gossypii. Biochem J. 2003;369(Pt 2):263-73.

19. Santos MA, Mateos L, Stahmann KP, Revuelta JL. Insertional mutagenesis in the vitamin B2 producer fungus Ashbya gossypii. Methods in Biotechnology. Microbial Processes and Products. New Jersey: Humana Press Inc; 2004

20. Livak KJ, Schmittgen TD. Analysis of relative gene expression data using real-time quantitative PCR and the 2(-Delta Delta C(T)) Method. Methods. 2001;25(4):402-8. doi:10.1006/meth.2001.1262.

21. Agren R, Liu L, Shoaie S, Vongsangnak W, Nookaew I, Nielsen J. The RAVEN toolbox and its use for generating a genome-scale metabolic model for Penicillium chrysogenum. PLoS Comput Biol. 2013;9(3):e1002980. doi:10.1371/journal.pcbi.1002980. 